\title{
Logistische Materialvorhaltung der Polytraumaversorgung im Schockraum aus radiologischer Sicht
}

\author{
Thomas Grieser
}

\section{Polytraumaversorgung am Universitäts- klinikum Augsburg in Zahlen - Überblick}

Im zurückliegenden Jahr 2018 wurden am Klinikum Augsburg, das über ein Level-1-Traumazentrum verfügt, insgesamt 663 Patienten gemäß Auswertung unseres radiologischen Leistungsregisters einem sog. Schockraum-CT zugeführt. Diese hohe Zahl - immerhin fast 2 Patienten pro Kalendertag - inkludiert allerdings alle Patienten, die auch aus anderen Gründen eine sog. „Polytraumaspirale“ erhielten (z.B. unklare Auffindungssituation, Stürze mit unklarem Verletzungsmuster, nicht beurteilbare und unklar verletzte Alkoholisierte, internistische Notfallsituationen unklarer Ätiologie etc.).

Bezogen auf die tatsächlich polytraumatisierten Patienten waren es laut TraumaRegister ${ }^{\circledR}$-Auszug der DGU (Deutsche Gesellschaft für Unfallchirurgie) insgesamt 264 Schockraumpatienten, d. h. mehr als 2 Patienten an 3 Tagen, die 2018 in unser Haus kamen, einen ISS $\geq 16$ (Injury Severity Score) hatten und/oder auf Intensivstation verlegt und/oder in den OP verbracht wurden. Von diesen waren es wiederum 111 Patienten, die alleinig einen ISS $\geq 16$ aufwiesen. Von der Gesamtzahl dieser Schockraumpatienten erhielten etwa $85-90 \%$ der Patienten eine Ganzkörper-CT, wobei eine diesbezügliche Auswertung bislang allerdings noch nicht systematisiert erfolgte. Im Vergleich hierzu erhielten 2016 entsprechend den Angaben des TraumaRegisters bundesweit knapp 80\% der Traumapatienten eine Ganzkörper-CT [1].

\section{Räumliche Situation der Polytrauma- versorgung am Universitätsklinikum Augsburg und kurzer Überblick über das Ablaufprozedere}

Das Anfang der 80er-Jahre erbaute und 1983 in Betrieb genommene Großklinikum Augsburg mit seinen rund 1700 Betten bietet aus heutiger Sicht strukturell nicht mehr zeitgemäße und baulich leider nur beengte Voraussetzungen für ein modernes Schockraummanagement. Zwar verfügt der bodengebundene Transport polytraumatisierter Patienten über einen geradlinigen und ebenerdigen Zugangsweg von der Anfahrt zur zentralen
Schockraumübergabestelle, jedoch muss bereits der hubschraubergestützte Patientenweitertransport im Hause zum einen allgemeine Publikums- und Patientenwege nutzen (die nur ausnahmsweise absperrbar sind), zum anderen weisen diese Wege enge Kurven und Kehren auf, die mit vollem Wiederbelebungsequipment nur verzögert passierbar sind.

Das Klinikum Augsburg verfügt über 3 nominale Schockräume (SR), deren Flächengröße mit ca. 26-34,5 m² zwar gerade noch den Leitlinienanforderungen genügt [2], aber allgemein als zu beengt angesehen werden muss (empfohlen 40-60 $\mathrm{m}^{2}$ nach [33]). Alle 3 Räume befinden sich zentral innerhalb der radiologischen Abteilung und sind sowohl von der Schockraumübergabestelle aus als auch von der Seite der Radiologie direkt erreich- und bedienbar. Je nach Ausstattungsniveau sind diese 3 Schockräume qualitativ abgestuft (SR 1-3) und werden auch in dieser Reihenfolge absteigend benutzt bzw. frequentiert.

Bezüglich der CT-Untersuchungsräume befindet sich das primäre, für die Polytraumaversorgung in erster Linie ausgestattete moderne CT in ca. $8 \mathrm{~m}$ Entfernung von der SRÜbergabestelle und ist von dort aus direkt anfahrbar. Obgleich es die Bezeichnung eines sog. „Schockraum-CT“ trägt, befindet sich dieses nicht im Schockraum! Gemäß dem Augsburger 2-Raum-Konzept der Polytraumaversorgung ist dieses $\mathrm{CT}$ weder räumlich noch ausstattungsmäßig für eine Akutintervention ausgelegt (Ausnahmeindikationen: laufende oder akute Reanimation, Thoraxdrainage bei Spannungspneumothorax, evtl. akute externe Blutungskontrolle). Prinzipiell wird aber in der Literatur entweder ein möglichst schockraumnaher Standort des CT propagiert [3] oder aber eine Integration des CT in den Schockraum selbst favorisiert [4].

Die beiden weiteren Computertomografen der radiologischen Klinik befinden sich in ca. $25 \mathrm{~m}$ bzw. immerhin $85 \mathrm{~m}$ Entfernung zur Übergabestelle und werden bei zeitgleich eintreffenden Notfall- und Schockraumpatienten oder beim Massenanfall von Verletzten (MANV) angefahren ( $\bullet$ Abb. 1).

Die zentrale Lage der Radiologie am Klinikum Augsburg erlaubt im Falle eines solchen Massenanfalles zudem die Öffnung ihrer zahlreichen umliegenden Untersuchungsräume auf ebengeschossigem Niveau, sodass in einem 


\section{VERWENDETE ABKÜRZUNGEN UND IHRE ERKLÄRUNGEN}

ALARA As low as reasonably achievable - Strahlenschutzgrundsatz bei der Anwendung ionisierender Strahlen beim Menschen

FAST Focused Assessment with Sonography for Trauma - rasch durchzuführende Suche nach freier intraperitonealer Flüssigkeit an definierten anatomischen Orten (subhepatisch, perisplenisch, parakolisch beidseits, retrovesikal [Douglas-Raum])

E-FAST Extended-FAST - bezieht das Perikardium (Herzbeuteltamponade) und den Pleuraraum ein (Hämato-/ Pneumothorax)

MANV MassenANfall von Verletzten - Großschadensereignis (Zugunglück, Massenkarambolage, schweres Busunglück, Bombenattentat u. a.) mit hoher Verletztenzahl $(n \geq 5)$ unterschiedlicher Schwere; situationsabhängig abgestufte und automatisierte Alarmierungsprozedur

REBOA Resuscitative endovascular Balloon Occlusion of the Aorta - dabei wird - mit oder ohne chirurgischer Leistenfreilegung - ein Ballonkatheter „blind“, d. h. ohne angiografische Kontrolle, über die Leistenarterie in die (infrarenale) Aorta abdominalis vorgebracht und geblockt, um die arterielle Blutzufuhr der unteren Körperhälfte bei schwerstem Blutverlust (z. B. bei schwerem Beckentrauma) bis zu einer definitiven (chirurgischen) Versorgung zu kontrollieren

RIS/PACS Radiologie-Informations-System/Picture archiving and Communication System - klinikeigene IT-Lösungen für die Patientendatenverwaltung und Bildarchivierung

ISS Injury Severity Score - anatomische Verletzungsgraduierungstabelle zur Abschätzung der Verletzungsschwere (erstmals 1974 eingeführt); ein ISS-Wert von > 15 entspricht einem Polytrauma; Werte zwischen 16 und 20 charakterisieren einen Schwerverletzten

SCIWORA Spinal Cord Injury without radiographic Abnormality - vor allem bei Kindern anzutreffendes Verletzungsmuster ohne röntgenologisch darstellbare Veränderungen bei jedoch mehr oder weniger schwerer diskoligamentärer Schädigung und/oder Rückenmarksverletzungen. Daneben gibt es aber auch eine Adult SCIWORA beim Erwachsenen.

solchen Falle gleichzeitig 5 weitere Untersuchungsräume sofort zur Verfügung stehen würden. Leichtverletzte und evtl. auch gehfähige Patienten könnten im Ausnahmefall zudem in die radiologischen Untersuchungsräume in die darüber gelegene Ebene verbracht werden (weitere 5 Räume unterschiedlicher radiologisch-apparativer Ausstattung).

Darüber hinaus sind insgesamt 2 moderne AngiografieSuiten vorhanden, von denen sich eine in unmittelbarer Nähe zur Schockraumübergabe befindet, die zweite allerdings räumlich entfernt im Obergeschoss; dort befindet sich auch die einzige radiologische Fluoroskopiemöglichkeit. Und schließlich können aber auch beide 1,5-TeslaMRT-Scanner (Siemens Avanto und Aera) im Untergeschoss bei besonderen Fragestellungen $(\triangleright$ Tab. 1 ) besetzt und angefahren werden.

Die zwar ebenerdig erreichbare, aber ca. 100 m entfernte Kinderradiologie verfügt über 3 nutzbare Radiologieräume (Röntgen + Sonografie) und ein MRT (3 Tesla Philips Ingenia); diese sind aber momentan kein fester Bestandteil des Augsburger Schockraummanagements.

Somit verfügt das Augsburger Großklinikum nominal über 3 Schockräume, ein sog. Schockraum-CT und eine Angiografie-Suite, die unter Routinebedingungen zur Versorgung Polytraumatisierter „24/7“, d.h. rund um

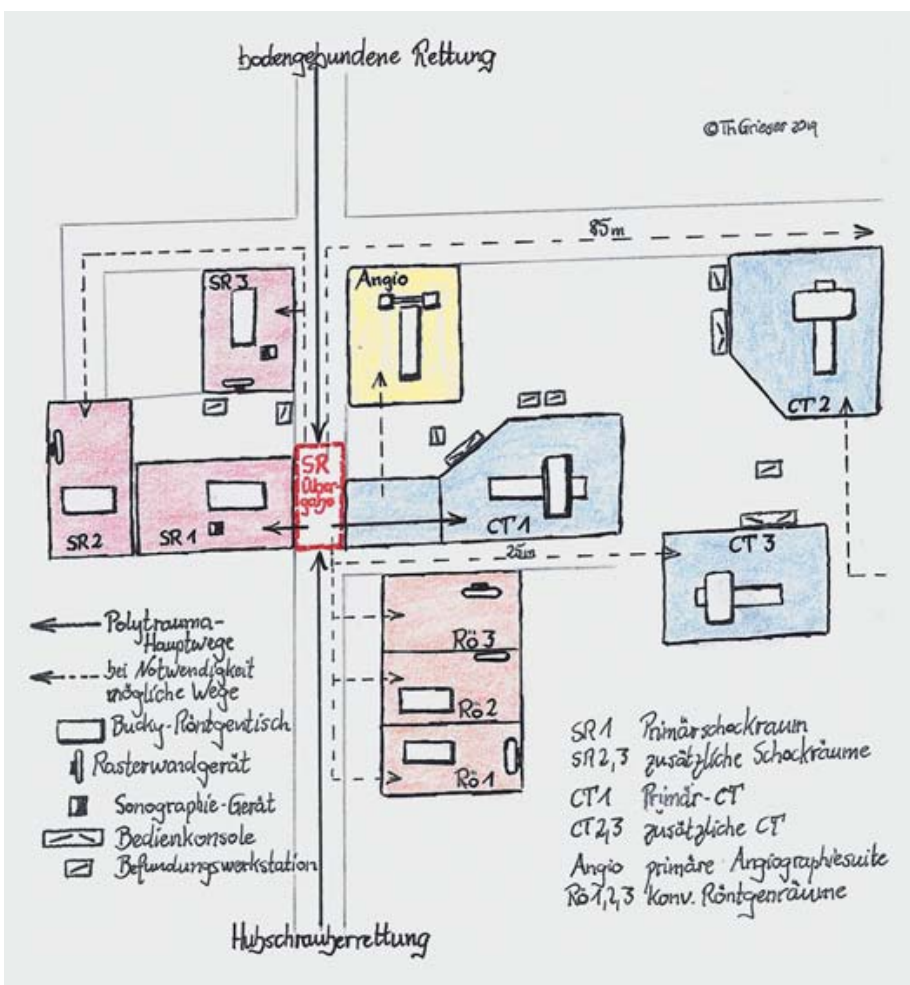

- Abb. 1 Nicht maßstabsgetreuer Lage- und Wegeplan der Augsburger Schockraumversorgung; alle Räumlichkeiten befinden sich ebenerdig und gehören zur radiologischen Abteilung. 
- Tab. 1 Indikationen zur Durchführung eines MRT in der postprimären Schockraumsituation. Voraussetzung ist ein kreislaufstabiler, unkritischer Patient.

\begin{tabular}{l|l|}
\hline $\begin{array}{l}\text { Szenario/Indikation } \\
\text { persistierende Bewusstlosigkeit, durch Trauma oder CCT nicht erklärbar }\end{array}$ & radiologische Fragestellung \\
\hline traumatische Dissektion (Karotis oder Vertebralis), im SR-CT unsicher & Dissektionsnachweis und mögliche frische Infarzierungen \\
\hline $\begin{array}{l}\text { akuter traumatischer Querschnitt } \\
\text { akute Querschnittssymptomatik, aber ohne radiologisches Korrelat im } \\
\text { SR-CT (z. B. Konus-Kauda-Syndrom) }\end{array}$ & $\begin{array}{l}\text { Beurteilung des medullären Status (Transsektion, Lazeration, Kontusion, Ein- } \\
\text { blutung); intraspinales Hämatom; Myelonkompression jedweder Ursache }\end{array}$ \\
\hline $\begin{array}{l}\text { akutes peripheres neurologisches Defizit } \\
\text { mit Myelonkompression }\end{array}$ \\
\hline
\end{tabular}

Tab. 2 Auswahl radiologisch-vaskulärer Interventionsmöglichkeiten in einer Akutsituation (traumatisch; anderweitige lebensbedrohliche Gefäßsituationen) [54].

\begin{tabular}{|c|c|}
\hline Lokalisation & radiologische Interventionsoptionen \\
\hline Aorta (1) & $\begin{array}{l}\text { Stentung von Typ-B-Dissektionen; geco- } \\
\text { verte Überstentung von Aortenrupturen }\end{array}$ \\
\hline Aorta (2) & $\begin{array}{l}\text { REBOA-Verfahren bei ansonsten unbe- } \\
\text { herrschbarer Blutungssituation der unte- } \\
\text { ren Körperhälfte/des Beckens einschl. } \\
\text { Beine (schwere Beckenquetschung, trau- } \\
\text { matische Amputation; Gefäßschussver- } \\
\text { letzungen) }\end{array}$ \\
\hline Milz & $\begin{array}{l}\text { Embolisation akuter Milzblutungen (Milz- } \\
\text { parenchymlazerationen) oder Ausschal- } \\
\text { tung von Milzarterienaneurysmata }\end{array}$ \\
\hline Nieren & $\begin{array}{l}\text { Embolisation von hämodynamisch wirk- } \\
\text { samen aktiven Nierenblutungen (retro- } \\
\text { peritoneal, Hohlsystem) }\end{array}$ \\
\hline $\begin{array}{l}\text { Gastrointes- } \\
\text { tinaltrakt }\end{array}$ & $\begin{array}{l}\text { Embolisation akuter und aktiver Magen-/ } \\
\text { Darmblutungen (Ulkusblutung, Diver- } \\
\text { tikelblutung, Angiodysplasieblutung) }\end{array}$ \\
\hline $\begin{array}{l}\text { periphere } \\
\text { Arterien }\end{array}$ & $\begin{array}{l}\text { Embolektomie/Angioplastie/Stentung } \\
\text { bei akuter Ischämie, Dissektion oder } \\
\text { traumatischen Pseudoaneurysmata; Em- } \\
\text { bolisation blutender peripherer Arterien- } \\
\text { äste (z. B. Profundaäste am Oberschen- } \\
\text { kel, egigastrische Gefäße, Beckenarte- } \\
\text { rien, Interkostalarterien) }\end{array}$ \\
\hline venös & $\begin{array}{l}\text { schwierige peripher-venöse Zugangs- } \\
\text { verhältnisse, blutende lliakalvenen; Cava- } \\
\text { filterinsertion }\end{array}$ \\
\hline
\end{tabular}

die Uhr, verfügbar sind. In Übereinstimmung mit der Literatur sind es die nachfolgenden beiden radiologischen diagnostisch-therapeutischen Modalitäten, die von ganz zentraler Bedeutung für ein funktionierendes Schockraummanagement sind $[41,42]$ :

1. durchgängige Verfügbarkeit eines modernen diagnostischen Schockraum-CT mit allen geeigneten und schnellen Nachbearbeitungsalgorithmen

2. ebenfalls durchgängige Verfügbarkeit einer radiologisch-angiografischen Interventionsfähigkeit (
Letzteres ist an unserer radiologischen Klinik „24/7“ gewährleistet und außerhalb der Regelarbeitszeit als Rufbereitschaft organisiert, wobei der telefonisch alarmierte Interventionalist sich bereits vorab teleradiologisch über die aktuelle Situation des Patienten (zumeist anhand des diagnostischen Kontrastmittel-CT) informieren kann, um Absprachen mit den zuständigen klinischen Partnern treffen zu können. Für sein Eintreffen bzw. Eingreifen vor Ort wurde eine 30-Minuten-Karenz vereinbart.

In diesem Zusammenhang soll an die Tatsache erinnert werden, dass die Mortalitätsrate bei hämodynamisch instabilen Patienten um immerhin 47\% je Stunde Verzögerung effektiver Maßnahmen steigt [43]. Gleichzeitig sei aber auch angemerkt, dass diese Mortalitätsrate nicht gleichzusetzen ist mit der „golden-hour-of-shock“-Regel [44], deren Gültigkeit in der jüngeren Literatur zunehmend hinterfragt wird [45] und stattdessen die Anpassung der Rettungskette an das Verletzungsmuster sowie an die Gegebenheiten am Unfallort zunehmend in den Vordergrund gestellt werden [46].

Im MANV-Fall sind zusätzlich 5 weitere Röntgenräume, 2 weitere CT-Räume und ggf. eine weitere Angiografieeinheit requirierbar, was in unseren hausinternen MANVAblaufleitlinien verbindlich so hinterlegt ist. Diese räumlich-technische Vorhaltung sollte aber auch dringend für den „Ernstfall“ geprobt werden (Simulierung eines MANVSzenarios), was bei uns letztmalig 2011 in großem Umfang geschah (Einschaltung aller verfügbaren präklinischen und klinischen medizinischen Einsatzkräfte sowie Feuerwehr, Polizei und THW).

\section{Das radiologische Equipment in den Schockräumen des Universitätsklinikums Augsburg}

Je nach Ausstattung sind die 3 o.g. Schockräume qualitativ abgestuft: Der für die Polytraumaversorgung vollständig ausgerüstete, primäre Schockraum (SR 1) verfügt radiologischerseits über einen Bucky-Röntgentisch und 

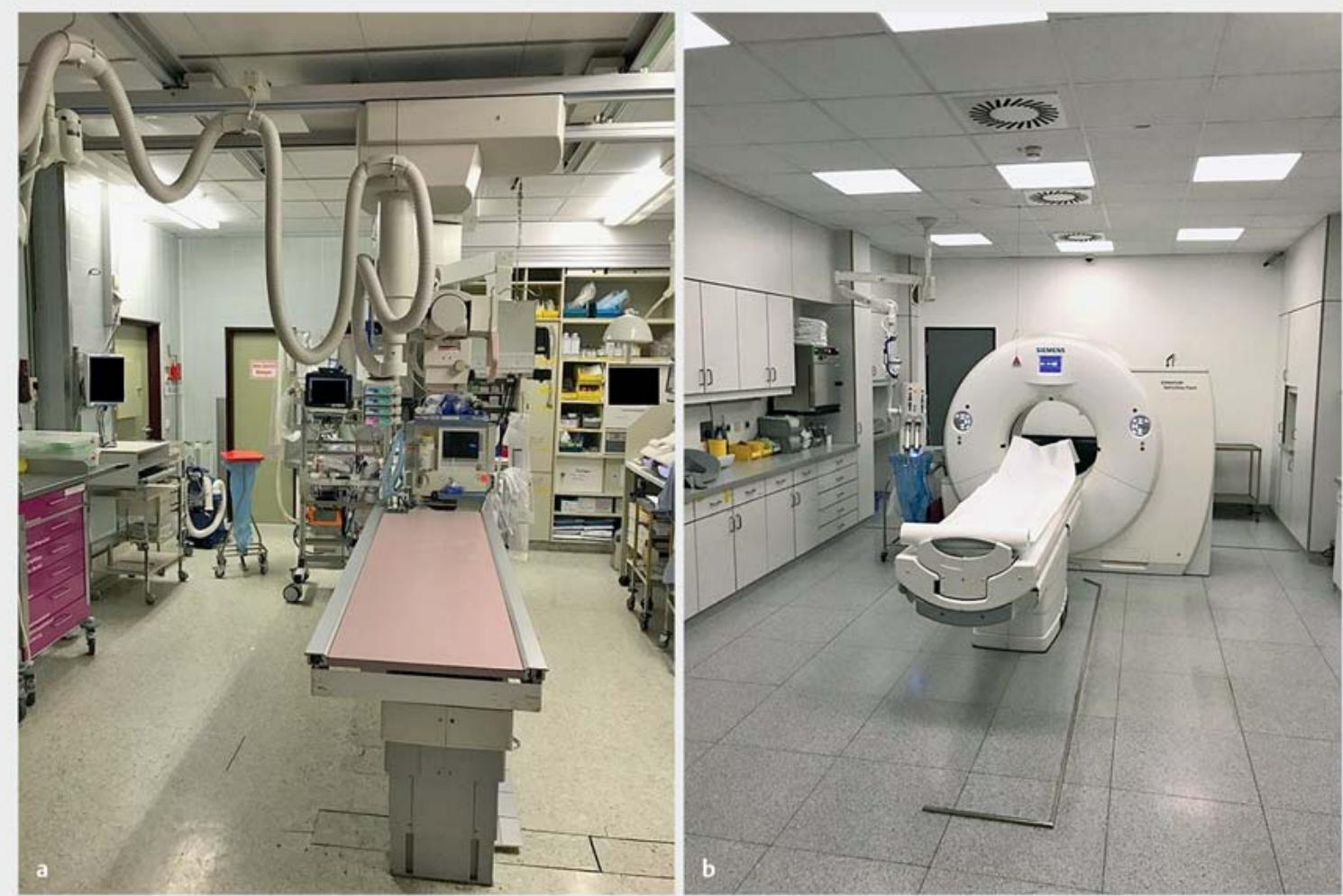

- Abb. 2 Augsburger 2-Raum-Konzept. Die linke Abbildung zeigt den primären Schockraum (SR 1) mit dem Bucky-Röntgentisch im Zentrum, dem anästhesiologischen Equipment dahinter und den zahlreichen Geräten, Instrumenten und Verbrauchsmaterialien zu beiden Seiten, wodurch sich die Bewegungsmöglichkeiten erheblich einschränken (Pfeile). Die rechte Abbildung zeigt das sog. Schockraum-CT, das allein in einem größeren Raum stehend mit deutlich mehr Bewegungsfreiheit untergebracht wurde.

ein permanentes Ultraschallgerät ( $\bullet$ Abb. 2 ); der sekundäre Schockraum (SR 2) sowohl über einen Bucky-Röntgentisch als auch ein Rasterwandgerät (RWG; das Ultraschallgerät kann durch eine Verbindungstür bei Bedarf rasch von SR 1 in den SR 2 verbracht werden) und der tertiäre Schockraum (SR 3) besitzt neben Bucky-Röntgentisch und RWG ein 2. permanentes Ultraschallgerät, verfügt aber nur über sehr limitierte notfallmedizinische Materialvorhaltungen.

Die genannten Räume sind einheitlich mit modernen digitalen Flachdetektoren ausgerüstet, die eine Sofortröntgenbilderstellung mittels Funkübertragung an die Bearbeitungskonsole erlauben, erheblich dosisreduziert arbeiten und kassettenfrei einfach zu handhaben sind. Alle Röntgeneinheiten sind vollständig digitalisiert mit dem RIS-PACS-Befundungssystem verbunden.

Die beiden permanenten, mobilen Ultraschallgeräte erlauben einen sofortigen und unmittelbaren Einsatz am polytraumatisierten Patienten durch den Radiologen des Schockraumteams im Rahmen des FAST- bzw. E-FASTKonzeptes [5,6]. Beide Geräte verfügen jeweils über einen 5-/7,5-MHz-Konvexschallkopf für die Abdominalso- nografie sowie über einen 10- bzw. 12-MHz-Linearschallkopf für oberflächliche, pleurale und vor allem kleinkindliche Darstellungen. Beide Geräte sind ebenfalls an das RIS-PACS-System angebunden.

Das diagnostische „Arbeitspferd“ hingegen stellt das sog. Schockraum-CT dar (siehe $>$ Abb. 2): Diese Bezeichnung ist insofern irreführend, als dass es sich nicht im Schockraum befindet, sondern räumlich getrennt davon aufgestellt ist (Augsburger 2-Raum-Konzept im Schockraummanagement). Es handelt sich um einen modernen $2 \times 128$-Zeilen-CT-Scanner der Fa. Siemens (Siemens CT Definition Flash) mit einer maximalen Scanlänge von $200 \mathrm{~cm}$ (Mindeststandard 16-Zeilen-CT It. S3-Leitlinie). Zudem ist die automatisierte Gabe vorgewärmten Kontrastmittels über einen maschinellen Hochdruckinjektor, der stets befüllt einsatzbereit zur Verfügung steht, routinemäßiger Standard, was insbesondere für CT-Angiografien und Perfusions-CT (für die Schlaganfallsdiagnostik) obligat ist.

In der direkt benachbarten Angiografieeinheit steht eine modernisierte digitale Subtraktionsangiografieanlage (Allura Xper der Fa. Philips), mit der alle gängigen Notfall- 


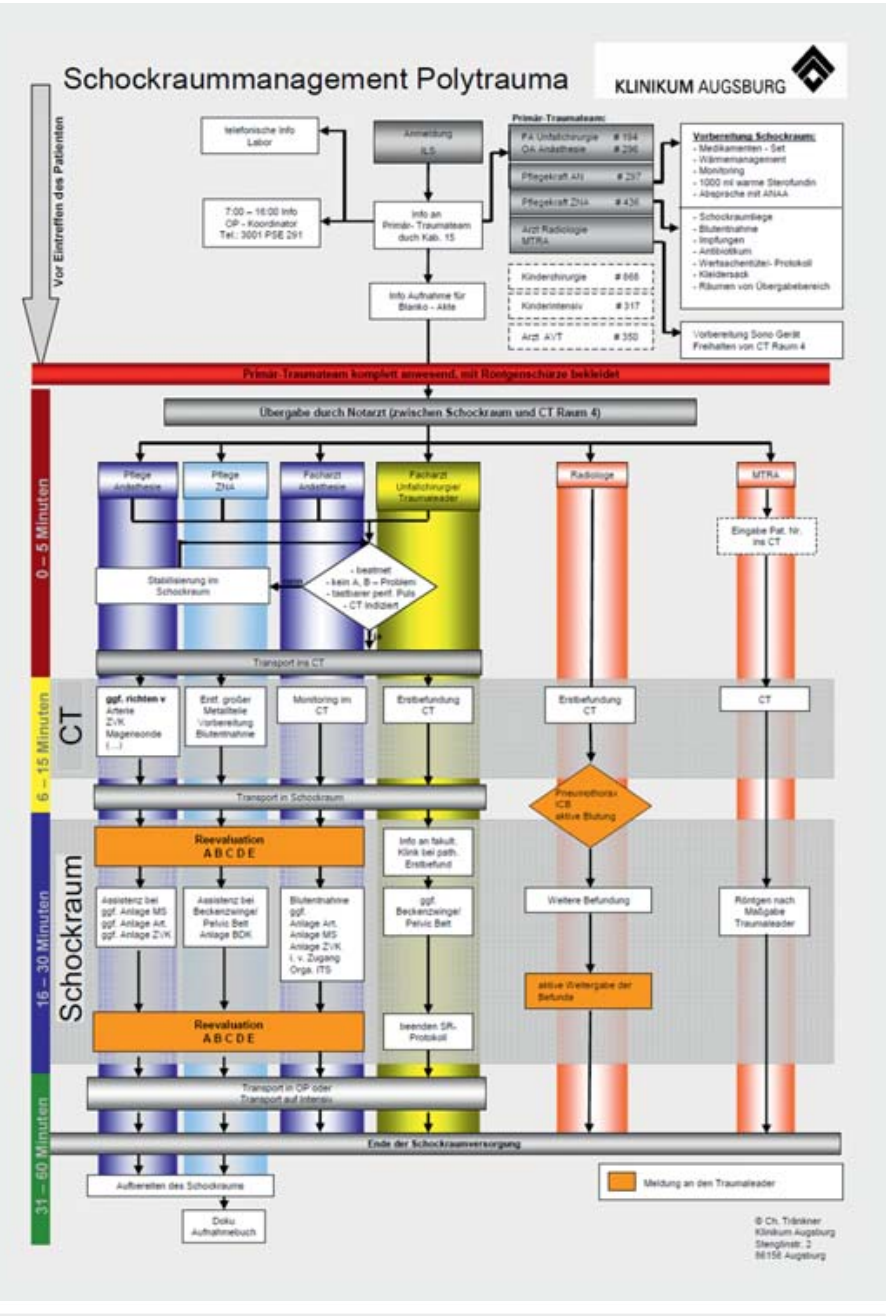

- Abb. 3 Augsburger Schockraumalgorithmus. Quelle: Ch. Tränkner, Augsburg.
Definition AS20, Scanlänge $197 \mathrm{~cm}$ ) sowie ein Multidektor-16-Zeilen-CT-Scanner der Fa. General Electric (GE BrightSpeed Elite, Scanlänge $160 \mathrm{~cm}$ ) älterer Bauart

An den CT-Konsolen und Arbeitsplätzen sind - an die jeweiligen CT-Scanner - angepasste und spezialisierte Protokolle zur Durchführung sog. Polytraumaspiralen einschl. ihrer Nachbearbeitungsalgorithmen hinterlegt, was den sofortigen und verzögerungsfreien Einsatz dieser Geräte im Bedarfsfalle erlaubt. Momentan sind an allen 3 CT-Geräten lediglich je ein geräteadaptiertes Standardprotokoll einheitlich hinterlegt, um stabile und individuell störunanfällige Bedingungen zu gewährleisten. Unter Dosisreduktionsgesichtspunkten wäre allerdings eine mehr klinisch adaptierte Untersuchungsstrategie mit 2 (oder mehreren) Untersuchungsprotokollen durchaus sinnvoll, wie jüngst vorgeschlagen [9]. Allerdings verfügen wir über ein speziell adaptiertes, dosisreduziertes Ganzkörper-CT-Protokoll für Kinder (hinterlegt als sog. „Kinderprotokoll“). Ebenso können an modernen (T-Scannern iterative Rekonstruktionsalgorithmen zum Einsatz gebracht werden, die ihrerseits zur weiteren Dosisreduktion führen [49].

Die Nutzbarkeit der 2. Angiografieeinheit hingegen ist im Bedarfsfall prinzipiell gegeben (Artis zee der Fa. Siemens). Wegen umfangreicher präinterventioneller Vorbereitungen (z. B. Narkose) spielt hierbei deren entfernte Lokalisation eine eher untergeordnete Rolle. Die Nutzbarkeit der gleichfalls räumlich entfernten radiologischen Fluoroskopiemodalität hingegen spielt im Schockraummanagement nahezu keine Rolle, zumal dieser Raum für intensivmedizinische Überwachungen ungeeignet ist.

\section{Radiologischer Workflow in der Polytraumaversorgung}

lisationen, Anlage gecoverter Stents bzw. Stentgrafts) durchgeführt werden können. Eine noch junge Sonderform der Blutungskontrolle schwerstverletzter Patienten im hämorrhagischen Schock stellt das sog. invasive REBOA-Manöver dar [8]. Das zugehörige Instrumentarium wird neuerdings gesondert im primären Schockraum vorgehalten und soll von einem speziell trainierten, interprofessionellen Schockraumteam im Bedarfsfall ohne angiografische Unterstützung im Schockraum zum Einsatz gebracht werden.

Beim erweiterten Schockraumbedarf, z.B. zeitgleiches Eintreffen zweier oder mehrerer Polytraumapatienten oder eines Schlaganfallpatienten, stehen folgende radiologische Equipments zur Verfügung:

- 2 weitere volldigitalisierte Bucky-Arbeitsplätze und 3 digitalisierte RWG in insgesamt 3 Röntgenräumen

- 2 weitere CT-Scanner: ein moderner Multidetektor20-Zeilen-CT-Scanner der Fa. Siemens (Siemens CT
Dieser schreibt zunächst vor, dass der Radiologe (in fortgeschrittener Ausbildung [Facharztstandard] oder Facharzt) essenzieller und obligater Bestandteil des Schockraumteams ist, was impliziert, dass der Radiologe bereits beim Eintreffen und der mündlichen Übergabe des Schockraumpatienten anwesend ist [10]. Dies erleichtert ihm die unmittelbare Koordination und ermöglicht die fachlich dedizierte Anweisung radiologischer Untersuchungen sowohl in eigener Regie (z. B. Sonografie) als auch die Instruktion der MTRA (z. B. Röntgen, CT) bzw. der für die Modalität zuständigen radiologischen Kolle- 

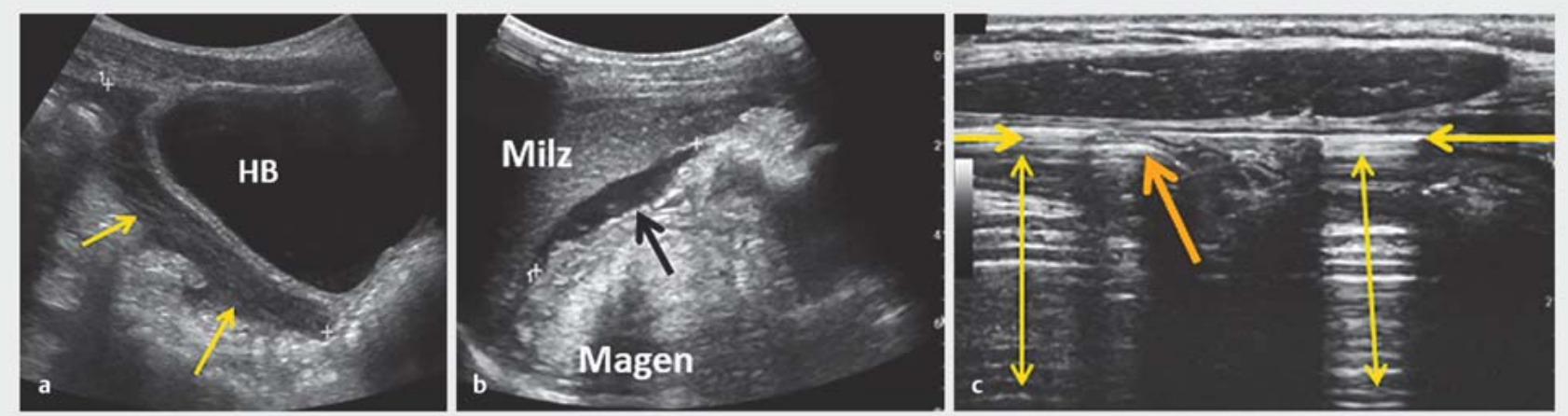

- Abb. 4 5-jähriger Junge, schwerer Frontalzusammenstoß. a Freie Flüssigkeit retrovesikal („Douglas-Raum“). b Freie Flüssigkeit perisplenisch. c Sonografischer Nachweis freier intraperitonealer Luft: subperitoneale Luftreflexe (gelbe Pfeile) mit typischen Reverberationsreflexen (Doppelpfeil); CAVE: diese Reflexe sind nicht mit Darmluftreflexen zu verwechseln (grüner Pfeil).

gen (interkollegiale und interprofessionelle Informationsweitergabe). Missverständnisse und Informationsverlust sollen so zumindest minimiert werden [11].

An der Schockraumübergabestelle fällt dann auch die prinzipielle Entscheidung über die „dichotome Prozedurentrennung":

1. hämodynamisch instabile Patienten und Patienten mit einem sog. A- und/oder B-Problem (Luftwegs-/Beatmungsproblem) werden zunächst zur unmittelbaren Wiederherstellung bzw. Stabilisierung und Aufrechterhaltung der Vitalfunktionen in den Schockraum verbracht

2. alle anderen Patienten gelangen unverzüglich (d.h. ohne weitere Maßnahmen) in das Schockraum-CT, Schockraum und Schockraum-CT sind räumlich getrennt! ( Tab. 3)

Die FAST- oder E-FAST-Sonografie wird durchgeführt, wenn der Patient prinzipiell nicht, zunächst nicht oder nicht sofort in das Schockraum-CT verbracht werden soll oder kann ( $\mathbf{A b b}$. 4). Deren Durchführung obliegt dem Radiologen; die Befundmitteilung erfolgt unmittelbar und zunächst mündlich an das Schockraumteam bzw. deren Trauma-Leader. Die FAST-Prozedur besitzt dabei eine hohe Spezifität (bis zu 100\%) bei einer allerdings deutlich niedrigeren Sensitivität (28-100\%) [12].

Entscheidend aber bleibt, dass jedwede Diagnostik effektiv und zielgerichtet ohne vermeidbaren Zeitverzug zur therapierelevanten Diagnose führt, um unverzüglich eine definitive Therapie einleiten zu können: Jede 3-minütige Verzögerung erhöht das Mortalitätsrisiko um $1 \%$ (für stumpfes Bauchtrauma) [38]!

Gleichzeitig (situationsabhängig, aber auch zeitlich abgesetzt) können ausgewählte Röntgenuntersuchungen im
- Tab. 3 Augsburger Schockraumindikationsliste zur Durchführung eines SR-CTs (Ganzkörper- oder Teilkörper-CT).

\section{Schockraumindikationsliste}

1. Sturz aus mehr als $3 \mathrm{~m}$ Höhe

2. Explosionsverletzungen

3. Einklemmung/Verschüttung

4. Herausschleudern aus dem Fahrzeug

5. Tod eines Mitfahrers

6. Motorrad- und Autounfall mit höherer Geschwindigkeit

7. Fußgänger/Radfahrer angefahren

8. Unfälle mit Pferden und Rindern (Großvieh; Reitunfälle, Hornverletzungen u.a.)

9. bewusstseinseingetrübter Patient mit Kopfverletzung

10. Schwerstkranke mit mutmaßlich akutem operativen und/oder interventionellen Handlungsbedarf

* SR-Indikation ist quasi gleichbedeutend mit SR-CT-Indikation

Schockraum erfolgen (Thoraxübersicht a.-p., HWS seitlich, Beckenübersicht a.-p.; $>$ Abb. 5).

Auch diese werden unmittelbar vom Radiologen noch an der Konsole begutachtet und zunächst mündlich kommuniziert. Bei beiden Untersuchungsmodalitäten im Schockraum (FAST/E-FAST und Röntgen) geht es vor allem um die Detektion potenziell lebensgefährlicher Pathologien (z. B. reichlich freie intraabdominale Flüssigkeit - Hämaskos, Spannungspneumothorax, Fehllagen eingebrachten Fremdmaterials) oder zügig zu therapierender Knochenpathologien (z. B. Luxationen, groteske Frakturfehlstellungen; $>$ Tab. 4).

Hierfür werden laut Literaturangaben ca. 15 min veranschlagt, was sich auch mit unseren Erfahrungen deckt [13]. Nach einer rezenten Vergleichsstudie (REACT-2) bleibt es unklar, ob der alleinige Einsatz des GanzkörperCT der Stufendiagnostik tatsächlich überlegen ist: Bezo- 

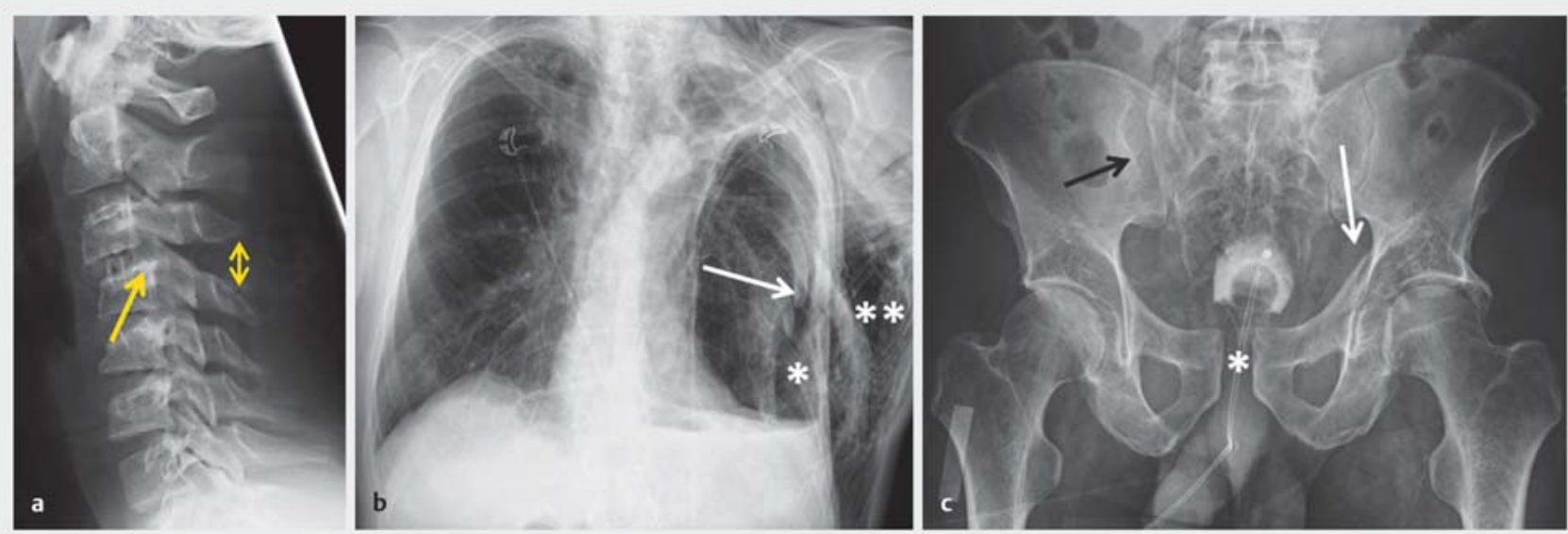

- Abb. 5 a 14-jähriger Junge, Sturz vom Baum: Flexionstrauma (Doppelpfeil) mit traumatischer Anterolisthese (Pfeil). b 58-jähriger Mann, schwerer Treppensturz: instabile Rippenserienfraktur links (Pfeil) mit Pneumothorax $\left({ }^{*}\right)$ und Weichteilemphysem $\left({ }^{* *}\right)$. c 59-jähriger Mann, Frontalcrash mit einem Geisterfahrer: Symphysensprengung (Open-Book-Verletzung; *), ISG-Sprengung rechts (schwarzer Pfeil) und Azetabulumfraktur links (weißer Pfeil).

- Tab. 4 Übersicht über mögliche radiologische Untersuchungsmodalitäten im Schockraum außerhalb der Schockraum-CT-Untersuchung.

\begin{tabular}{|c|c|}
\hline $\begin{array}{l}\text { radiologische Moda- } \\
\text { lität im Schockraum }\end{array}$ & radiologische Beurteilungskriterien \\
\hline FAST-Ultraschall & $\begin{array}{l}\text { freie Flüssigkeit (perihepatisch, perisplenisch, } \\
\text { Unterbauch); grobe Organlazerationen (Leber, } \\
\text { Milz, evtl. Nieren); retroperitoneales Hämatom }\end{array}$ \\
\hline E-FAST-Ultraschall & $\begin{array}{l}\text { Mitbeurteilung Perikard (Perikardtamponade) } \\
\text { und Pleurahöhlen (Pneumothorax, Hämato- } \\
\text { thorax) }\end{array}$ \\
\hline $\begin{array}{l}\text { Röntgen HWS seitlich } \\
\text { (optimal bis HWK VII } \\
\text { bzw. bis zum zerviko- } \\
\text { thorakalen Übergang) }\end{array}$ & $\begin{array}{l}\text { Alignment?, Zwischenwirbelraum; HWK-Frak- } \\
\text { turen (vor allem tear-drop, Spondylophyten), } \\
\text { Dornfortsatzfrakturen; prävertebraler Weich- } \\
\text { teilschatten? }\end{array}$ \\
\hline $\begin{array}{l}\text { Röntgenthorax a.-p. } \\
\text { im Liegen }\end{array}$ & $\begin{array}{l}\text { (Spannungs-)Pneumothorax, (Komplett-)Ver- } \\
\text { schattung, Mediastinalshift, Mediastinalver- } \\
\text { breiterung; grob dislozierte Rippenfrakturen; } \\
\text { Enterothorax? }\end{array}$ \\
\hline $\begin{array}{l}\text { Röntgen Beckenüber- } \\
\text { sicht a.-p. }\end{array}$ & $\begin{array}{l}\text { Fraktur? und Ausmaß der Fraktur (instabil?), } \\
\text { Open-Book-Verletzung, Azetabulumfraktur, } \\
\text { traumatische Hüftluxation }\end{array}$ \\
\hline
\end{tabular}

gen auf die Mortalität ergibt sich kein Vorteil [14], allerdings verkürzt der primäre und ausschließliche CT-Einsatz die Zeit vom Eintreffen des Patienten bis zur Diagnosestellung [15]. Demzufolge besitzen zahlreiche Traumazentren lediglich nur noch ein Multidetektor-CT im Schockraum bzw. seiner näheren Umgebung, was in der Literatur aber durchaus kritisch gesehen wird [16].

Abgesehen von peripheren bzw. akralen Extremitätenverletzungen (z. B. undislozierte und unkomplizierte Fraktu- ren langer Röhrenknochen, Fingerfrakturen), beschränkt sich die röntgenologische Abklärung auf die 3 weiter o.g. röntgenologischen Darstellungen. Gelenkverletzungen (z. B. Hand- und Sprunggelenksfrakturen, Fußfrakturen, aber auch Ellenbogen- und Kniegelenksfrakturen) sollten - wenn immer möglich - bereits in die primäre Schockraum-CT-Abklärung inkludiert werden.

An dieser Stelle sei nochmals hervorgehoben, dass in bewusster Abgrenzung zu anderen Schockraumkonzepten $[7,48]$ in unserem Schockraum-CT (bis auf sehr wenige Ausnahmen) keinerlei interventionelle Prozeduren vorgenommen werden (dürfen); er dient damit ausschließlich einer sehr rasch durchzuführenden Diagnostik. Eine längere zeitliche Blockierung des Raumes soll hierdurch vermieden werden.

Die Schockraum-CT-Auswertung durch den Radiologen erfolgt 3-stufig ( Abb. 6) [54]:

1. simultane Beobachtung der Untersuchung an der Konsole durch den Radiologen und seine unmittelbare mündliche Übermittlung lebensbedrohlicher bzw. unmittelbar therapierelevanter Befunde (massive intrakranielle Blutung, Spannungspneumothorax, aktive [arterielle] Blutung); Zeitrahmen: 0-5 min [36]

2. anschließende, eingehende Durchmusterung aller verfügbaren axialen Schichtbilder und multiplanaren Rekonstruktionen an der Befundungs-Workstation durch den Radiologen mit anschließender strukturierter mündlicher Weitergabe aller relevanten Befunde an den Trauma-Leader im Zeitfenster des vereinbarten Time-outs nach 20 bis max. 30 min im Schockraum $(\vee$ Abb. 7) [11]; 
3. Detaillierte schriftliche radiologische Befunderstellung; mitunter verzögert oder nach nochmaliger Durchsicht und Diskussion auftretende relevante Befundänderungen werden dem Trauma-Leader mündlich mitgeteilt [37]. Zur Veranschaulichung komplexer Traumabefunde (z.B. dislozierte Rippenfrakturen, komplexe Wirbelkörper- oder Beckenfrakturen, dislozierte Extremitätenfrakturen mit Darstellung des Gefäßbezuges, unübersichtliche Hand- und Fußfrakturen; > Abb. 8) können auch spezielle bzw. adaptierte 3-D-Darstellungen generiert werden.

Dem Radiologen steht eine Workstation zur dezidierten Bildbetrachtung und Bildauswertung zur eigenen Verfügung; darüber hinaus kann er bei Bedarf zur weiteren Bildauswertung zusätzlich auf eine direkt mit dem CTScanner verbundene Server-Client-basierte Software zurückgreifen (Syngo.via ${ }^{\circledR}$ der Fa. Siemens) mit zahlreichen vorinstallierten Rekonstruktionstools (3-D-Rekonstruktionen in verschiedenen Darstellungsmöglichkeiten, automatisierte Rippenrekonstruktionen, übersichtliche Darstellung komplexer Mittelgesichtsfrakturen u.v.m.) [50-52]. Für die am Schockraumpatienten tätigen klinischen Partner steht eine separate Bildbetrachtungskonsole zur Verfügung.

Der vor Ort verantwortliche Radiologe besitzt die Fachund Sachkunde Notfallradiologie einschließlich CT. Unklare oder problematische Befundkonstellationen werden mit einem verantwortlichen oder dem diensthabenden radiologischen Oberarzt besprochen; dies gilt insbesondere für potenziell therapierelevante oder -verändernde Befunde. Eine nachlaufende, systematische Befundkon- trolle durch einen in der Polytraumadiagnostik erfahrenen radiologischen Fach- oder Oberarzt erfolgt spätestens am Folgetag und wird - sofern personalmäßig realisierbar - konsequent durchgeführt. ( $\triangleright$ Tab. 5) [34, 37].

Es gibt mehrere klinische Szenarien, die zum begründeten Abweichen vom hier dargelegten radiologischen Ablaufschema Anlass geben:

1. verunfallte oder anderweitig physisch traumatisierte Kinder und Jugendliche (16 Jahre und jünger)

2. Schwangere unterschiedlichen Gestationsalters

3. Patienten mit einer akuten, traumaassoziierten Querschnittssymptomatk (auch der Verdacht auf das Bestehen einer solchen bzw. einer unvollständigen Querschnittssymptomatologie)

Bei Kindern und Adoleszenten wird die Indikation - trotz adäquaten Traumas bzw. Traumamechanismus - zur CT wegen der bekannten hohen Strahlensensibilität des wachsenden Organismus besonders streng gestellt (ALARA-Prinzip) [17]. Die unkritische Anwendung der Ganzkörper-CT (GK-CT) bei Kindern wird daher mehrheitlich als problematisch angesehen und sollte durch eine sorgfältige Risikostratifizierung vermieden werden, zumal gezeigt werden konnte, dass der Einsatz der GK-CT nicht per se zu einer signifikanten Mortalitätssenkung und verbessertem Outcome führt [18-20], wodurch die sonografische Evaluation wiederum stark an Bedeutung gewinnt [21]. Eine erst jüngst veröffentlichte Studie konnte zeigen, dass bei Kindern die alleinige Beachtung des Traumamechanismus bzw. des Glasgow-Coma-Scales (außer beim Schädel-Hirn-Trauma) keine guten Prädiktoren der thorakoabdominalen Verletzungsschwere dar-

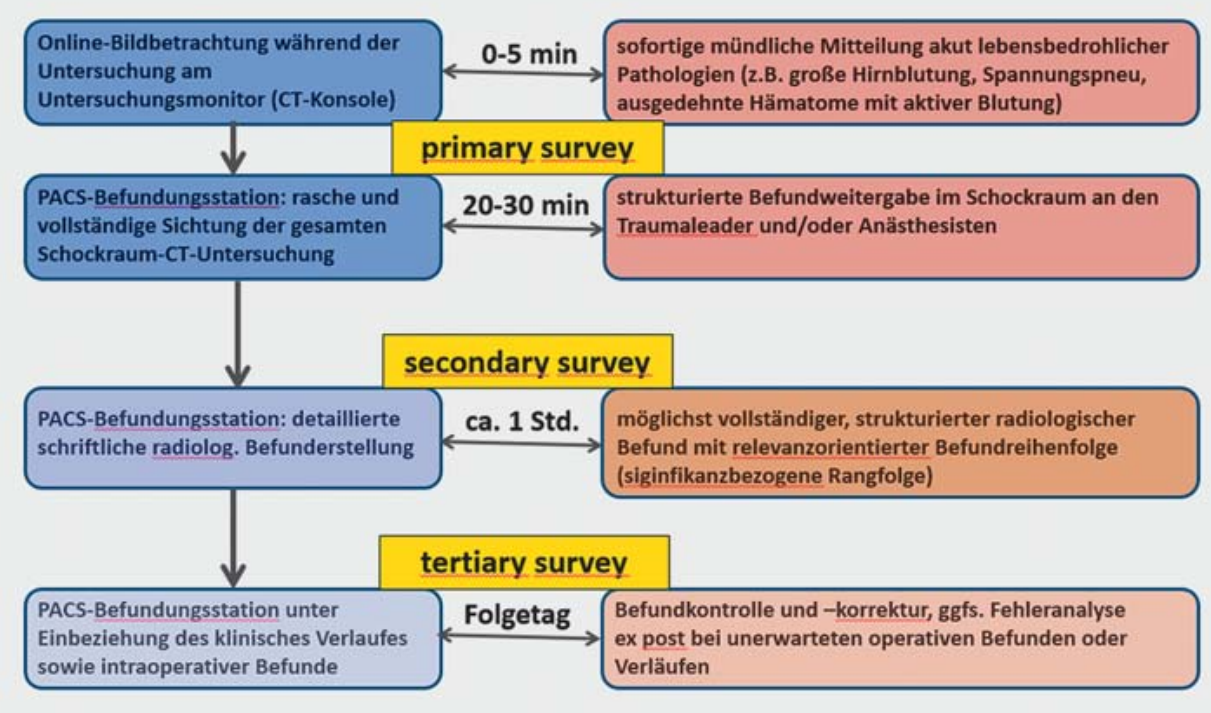

Abb. 6 Überblick über den radiologischen Befundungs-Workflow bei der Auswertung einer Schockraum-CT-Untersuchung. 


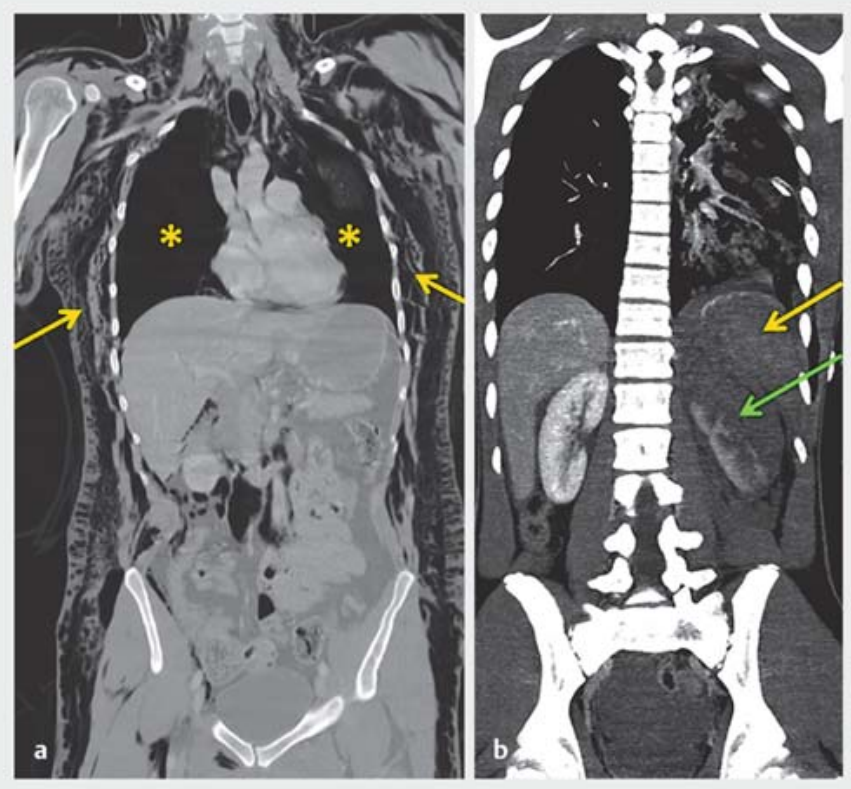

- Abb.7 Zwei junge verunfallte Männer. a Bilateraler Pneumothorax mit einem massiven ubiquitären Weichteilemphysem sowie freier intra-/ retroperitonealer Luft per continuitatem. b Vollständiger Milzgefäßstielabriss und subtotale Nierengefäßamputation links.

stellen, was die Indikation zum GK-CT bei Kindern einschränkt und den Stellenwert der FAST-Sonografie für abdominelle Verletzungen hervorhebt (siehe $>$ Abb. 4) [32].

Trotz i. d. R. guter Schallbedingungen im Kindesalter stellt die pädiatrische Notfallsonografie kritisch verletzter Kinder stets hohe Ansprüche an die Erfahrung des geübten radiologischen Sonografeurs: Schlechte Datenlage, hohe Heterogenität der kindlichen Verletzungsmuster und die nicht flächendeckend vorhandene hohe sonografische Expertise erlauben jedoch keine abschließende Empfehlung zum hervorgehobenen Einsatz der Sonografie beim kindlichen Trauma [22].

Polytraumatisierte Schwangere ( $\triangleright$ Abb. 9) - sofern bekannt - stellen nach wie vor eine besondere Herausforderung an die Bildgebung dar: Verletzungsmechanismus (z.B. Gurtverletzung über dem graviden Uterus) und klinische Schwere (bewusstlos, intubiert und beatmet) zwingen zu einem individuell angepassten diagnostischen Vorgehen, das sowohl die maternale Bedrohung durch das Trauma (z. B. hämorrhagischer Schock) als auch die spezifischen schwangerschaftsassoziierten Komplikationen (intrauteriner Fruchttod, Uterusruptur, Abruptio placentae u.a.m.) zu berücksichtigen hat [23-25].

Darüber hinaus spielt die Wahl der bildgebenden diagnostischen Modalität (Ganzkörper-CT vs. Teilkörper-CT,
- Tab. 5 Typischerweise übersehene Befunde, die bei der systematischen Befundnachschau identifiziert werden können [54].

\section{Häufig bzw. typisch übersehene Befunde in der initialen} CT-Schockraumbefundung

frontale und temporale Hirnparenchymkontusionen

Subarachnoidalblutung in der interpedunkulären Fossa bzw. in der Sylvi'schen Fissur

DAI (diffuse axonal injury): kleine stippchenförmige Blutungen perikalossal, an den Stammganglien und an der hemisphärischen Mark-Rinden-Grenze

Okzipitalkondylenfrakturen und knöcherne Ausrisse der Ligg. alaria

Karotiden- und Vertebralisdissektionen (vor a,llem. in Schädelbasisnähe)

Skapulafrakturen, mediale > laterale Klavikulaluxationen intraparenchymatöse Leber-/Milzlazerationen (KM-phasenabhängig)

Iliakalgefäßblutungen, vor allem venöse Gefäßverletzungen

Harnblasenrupturen (dran denken!)

Hohlorganperforationen ohne initialen freien Luftnachweis (deswegen geeignete Kontrolle bei klinischer Verschlechterung der Abdominalsymptomatik sinnvoll)

periphere Frakturen und Luxationen (Extremitäten einschl. Hände und Füße)

CT vs. Sonografie allein und/oder MRT) unter dem Aspekt des Strahlenschutzes eine wichtige Rolle: Im Falle eines GK-CT sollte die effektive Organdosis (Uterus) dokumentiert und von einem Medizinphysiker berechnet bzw. qualifiziert geschätzt werden (Stufe I-III), um das fetale Strahlenrisiko estimieren zu können [27]. Als kritischer Wert werden 20 mSv Uterusorgandosis angesehen, oberhalb derer genauere Dosisermittlungen empfohlen werden [47]. Dieser Wert kann im Rahmen eines Polytrauma-CT ohne Weiteres erreicht werden.

Die traumaassoziierte Querschnittssymptomatik zieht i.d.R. eine weitere MRT-Abklärung im Rahmen des Polytraumamanagements nach sich, sofern es sich um kreislaufstabile Patienten handelt und vor einer chirurgischen WS-Stabilisierung eine bildgebende Diagnostik zum Zustand diskoligamentärer Strukturen und des Myelons gefordert ist $[28,29]$. Auch hierbei spielen Kinder eine herausgehobene Rolle infolge radiologisch okkulter, schwerer WS-Verletzungen mit substanziellen neurologischen Defiziten, mitunter ohne erkennbare radiografische Verletzungsmuster (SCIWORA-Konzept) [30,31] Letzteres wird gelegentlich auch bei erwachsenen und älteren Unfallopfern angetroffen ( $\triangleright$ Abb. 10).

Ohne detailliert auf die vielfältigen Entscheidungsfindungen eingehen zu können, stimmen alle vorgenannten Szenarien darin überein, dass sie stets einer engen Ab- 


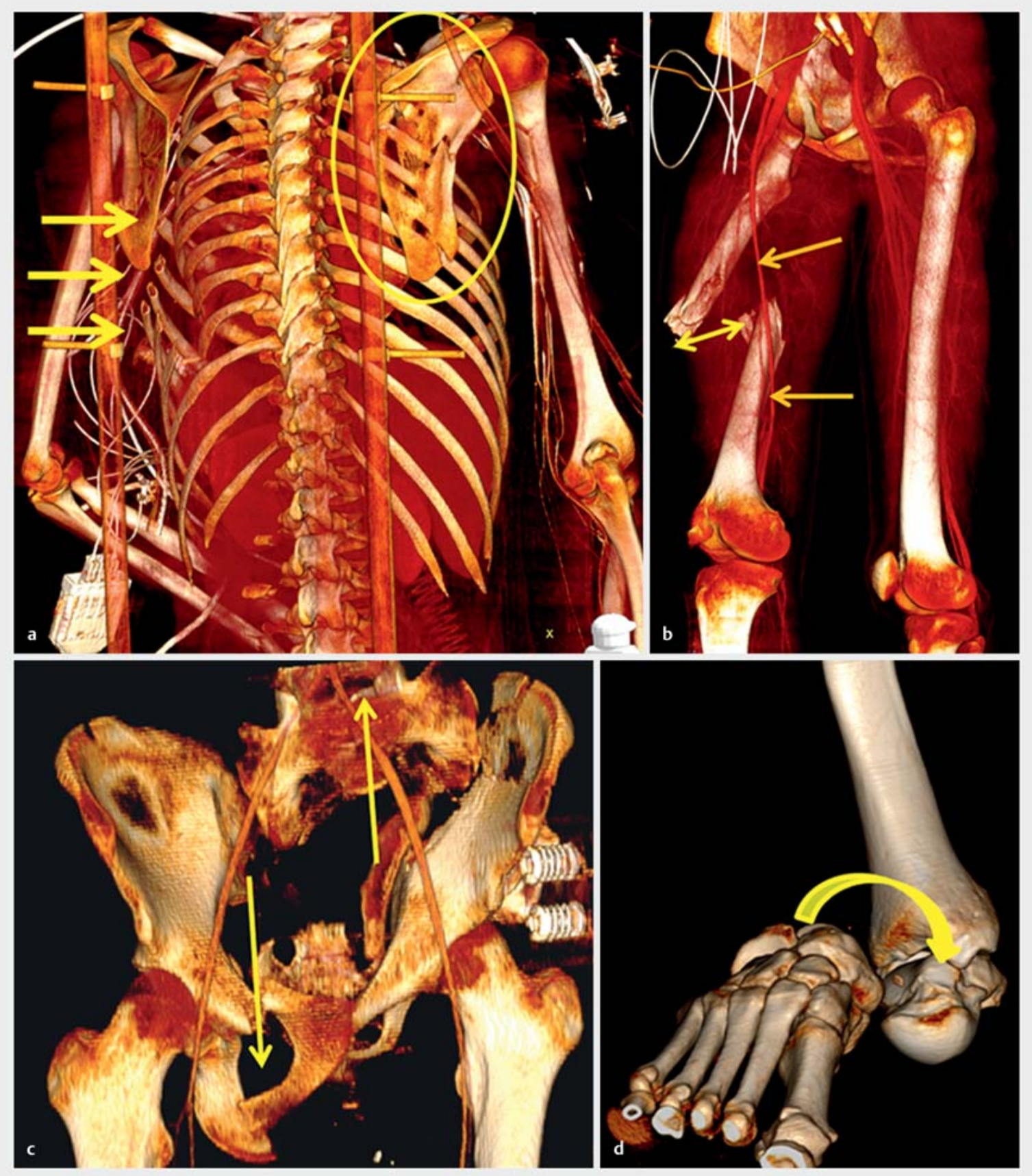

- Abb. 8 Dreidimensionale Volumendarstellung (volume rendering technique) zur Darstellung komplexer Traumapathologien. Links oben: dislozierte Rippenserienfraktur; rechts oben: dislozierte Femurfraktur in unmittelbarer Nähe zur A. femoralis superior; links unten: vertikal instabile Tile-C-Beckenringfraktur; rechts unten: Luxatio pedis sub talo.

stimmung mit den klinischen Partnern sowohl des Kernals auch des erweiterten Schockraumteams (Unfallchirurg [Trauma-Leader], Anästhesist; Neurochirurg/Neurologe, Thorax- und Abdominalchirurg, Gefäßchirurg, Kinderchirurg, Intensivmediziner, Gynäkologe bzw. Geburtshelfer, HNO-Kollegen und Kieferchirurgen u. a.) bedürfen [33, 53]. 


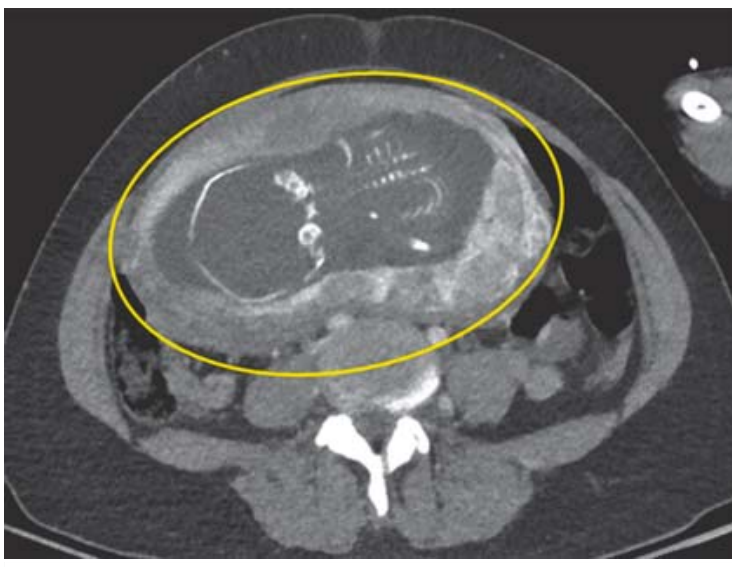

- Abb. 9 Schwer verunfallte, aber nicht bewusstlose afrikanische Emigrantin, Sprachbarriere, Schwangerschaft (24. SSW) daher nicht bekannt: CT ist dennoch aufgrund des Hochrasanztraumas indiziert gewesen.

\section{Vergleich des Augsburger Modells mit den Vorgaben der aktuellen S3-Leitlinie Polytrauma/Schwer- verletzten-Behandlung}

Wenngleich es durchaus räumliche und ausstattungstechnische Mängel und Unzulänglichkeiten gibt (siehe nächster Abschnitt „Ausblick ..."), so genügt das Augsburger Schockraummodell doch in seinen wesentlichen und primären Punkten jenen Anforderungen, die durch die aktuelle S3-Leitlinie Polytrauma/SchwerverletztenBehandlung vorgegeben sind [2]. Aus radiologischer Sicht bedeutet dies konkret Folgendes:

1. Die permanente und einsatzbereite Verfügbarkeit des Schockraum-CTs, die ständige Einsatzbereitschaft von mindestens 3 Röntgeneinheiten (Bucky-Tische) mit volldigitaler Röntgendetektorausstattung sowie die sofortige Einsatzbereitschaft von 2 Ultraschallgeräten in den Schockräumen ermöglichen den unmittelbaren und verzögerungsfreien Einsatz im Rahmen des Primary Survey.

2. Bezüglich der Anordnung des CT-Scanners außerhalb des Schockraumes modifiziert das Augsburger Modell die Empfehlungen der S3-Leitlinie, welche die Lokalisation des CT in unmittelbarer Nähe des Schockraumes ausdrücklich erlaubt.

3. Die räumliche Diversifikation Schockraum - CT-Raum impliziert auch eine geänderte Strategie hinsichtlich evtl. notwendiger Eingriffsprozeduren: Das Augsburger Konzept sieht eine strikte Trennung zwischen CTDiagnostik und Notfalleingriffen vor, die nahezu ausschließlich im voll ausgerüsteten Schockraum, nicht aber im CT stattfinden sollen. Reanimationsmaßnahmen bleiben hiervon natürlich unberücksichtigt, ebenso wie lebensrettende Sofortmaßnahmen (z. B. Thoraxdrainageanlage bei Spannungspneumothorax, hämorrhagische Herzbeuteltamponade); sie bilden aber die Ausnahme.

4. Die personelle Besetzung in der Radiologie sieht zum einen eine ständige ärztliche Anwesenheit vor Ort (auch nachts) vor, zum anderen aber auch eine permanente, mindestens 3-köpfige MTRA-Anwesenheit. Dieses Personal kann dabei geplant durch je einen Fach- oder Oberarzt (Interventionalist) und/oder eine (n) MTRA aus dem Rufdienst erweitert werden.

5. Der radiologische Workflow von der Befunderhebung (Scan) über die Befundweitergabe bis zum definitiven radiologischen Befund entspricht den Vorgaben der Leitlinie. Ihre strikte Anwendung und zeitliche Stringenz hängt allerdings erheblich vom Ausbildungsstand und der praktischen Erfahrung des Radiologen in der Polytraumabeurteilung ab.

6. Die Entscheidung zur akut angiografischen Intervention (z. B. Blutungsembolisation) wird zumeist noch in der Schockraumphase des Patienten getroffen und nach konsentierter Entscheidung im Traumateam vor Ort umgesetzt. Als günstig hierfür erweist sich die unmittelbare räumliche Nähe der Angiografieeinheit zum Schockraum bzw. zum Schockraum-CT; eine sofortige Verbringung des Patienten entweder direkt aus dem Schockraum (z. B. im Rahmen eines notwendigen REBOA-Manövers unter angiografischen Bedingungen) oder unmittelbar nach dem diagnostischen Schockraum-CT (z. B. Nachweis einer aktiven arteriellen Blutung mit oder ohne hämorrhagischen Schock) ist daher ohne Weiteres möglich ( $\boldsymbol{A} \mathbf{A b b} \mathbf{b} \mathbf{1 1}$ ). Sollte eine primär operative Versorgung zur Blutungskontrolle ineffektiv bleiben, wird ebenfalls die interventionell-radiologische Therapieoption in die Wege geleitet (postprimäre Phase).

7. Die MRT besitzt momentan am Klinikum Augsburg noch keinen fest zugewiesenen Platz im klinikeigenen Polytraumaalgorithmus, gleichwohl gibt es bereits eine Vielzahl von Empfehlungen bzw. umgesetzten Prozeduren bez. der Einbettung des MRT in ein primäres Schockraumkonzept $[39,40]$. Allerdings kommt der MRT in der sog. postprimären Versorgungsphase Schwerverletzter in unserem Hause insofern Bedeutung zu, als dass vor allem bei traumatischer Querschnittssymptomatik unterschiedlicher Ausprägung und Qualität eine MRT zügig - indikationsabhängig mitunter noch vor einer primären chirurgischen Versorgung - zum Einsatz gebracht wird ( $\bullet$ Tab. $\mathbf{6})$.

8. Die Diagnostik (poly-)traumatisierter und insbesondere kleiner Kinder $<6$ Jahren erfolgt unter Berücksichtigung der erhöhten Strahlensensibilität des kindlichen Organismus fallorientiert und individuell angepasst, wobei dem Ultraschall als Modalität der ersten Wahl ein hoher Stellenwert zukommt. Stets erfordert dieses Vorgehen neben einem hohen Maß an Verantwortung und Erfahrung eine enge Absprache mit den 

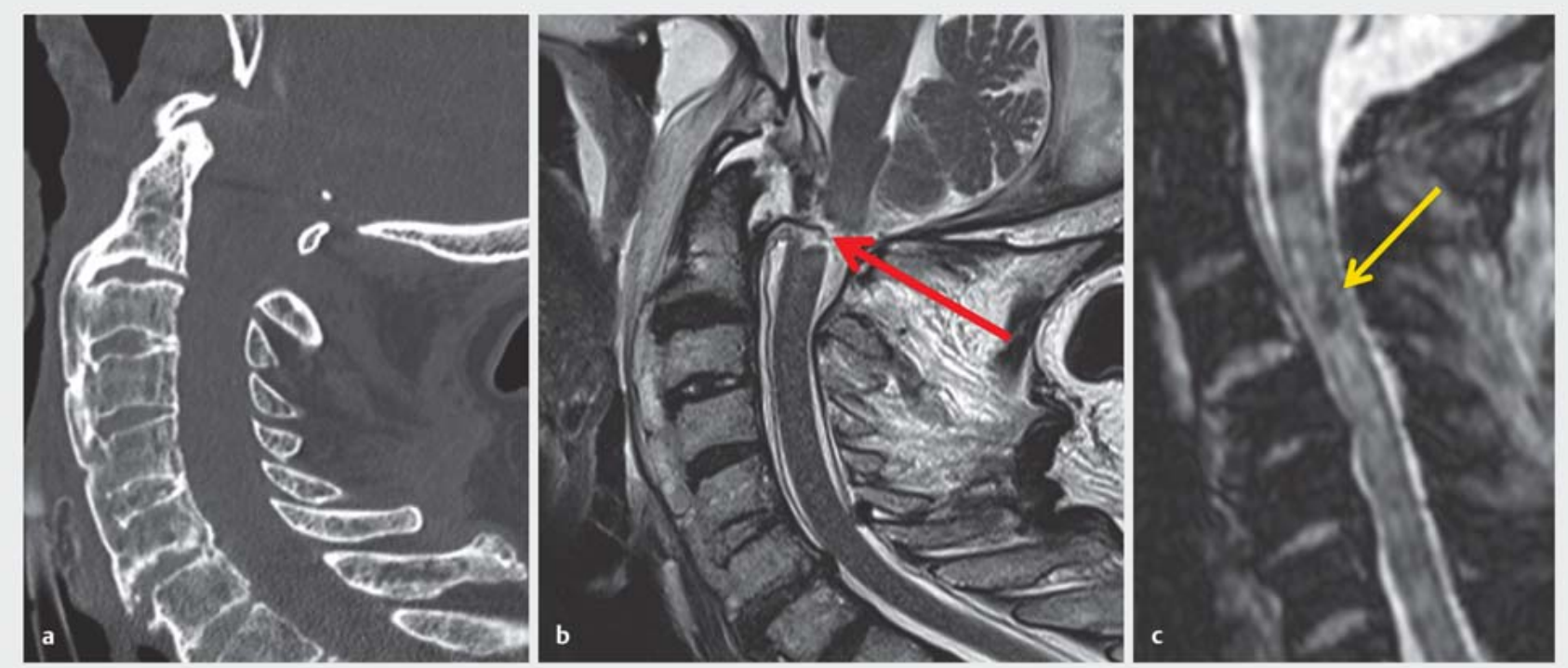

- Abb. 10 Adulte Form des SCIWORA. a, b 80-jähriger Mann, Treppensturz: röntgenologisch unauffällig, aber im MRT vollständige Myelontranssektion (Pfeil). c Anderer Patient, unauffällige Radiografie: Myelonhämorrhagie (Pfeil); beide Patienten hatten einen kompletten hohen Querschnitt.

weiterbehandelnden Kinderchirurgen bzw. intensivmedizinisch tätigen Pädiatern.

9. Gleiches gilt in analoger Weise für das diagnostische Prozedere verunfallter schwangerer Patientinnen unter Hinzuziehung erfahrener Gynäkologen bzw. Geburtshelfer. Sollte dennoch eine GK-CT-Abklärung erforderlich werden, so erfolgt anschließend eine Organdosisabschätzung oder -berechnung für den Uterus durch den Medizinphysiker.

\section{Ausblick auf wünschenswerte logisti- sche und materielle Verbesserungen in radiologischer Hinsicht}

Um der steigenden Frequenz in der Versorgung polytraumatisierter Patienten am Augsburger Universitätsklinikum gerecht zu werden, stünde eine komfortablere räumliche Ausstattung ganz im Vordergrund. Die oben skizzierten beengten Verhältnisse stellen nicht nur ein Ärgernis für die handelnden Akteure dar, sondern entwickeln sich auch zunehmend zum Problem einer modernen und fortgeschrittenen Schockraumversorgung. Seiner Lösung stehen natürlich die baulichen Gegebenheiten entgegen, die nicht bzw. nicht ohne substanzielle strukturelle Umbaumaßnahmen lösbar sind. Eine Umsetzung derartig umfangreicher Baumaßnahmen ist derzeit nicht in Sicht.

Deshalb wurden und werden Vorschläge unterbreitet, unterhalb der Ebene groß angelegter baulicher Maßnahmen bereits mit geringeren Mitteln Änderungen vor-
- Tab. 6 Typische Indikationen zur Durchführung einer spinalen MRT (primär oder postprimär) [39].

\section{Hauptindikationen zur MRT beim traumatischen spinalen Notfall (Auswahl)}

1. jeder traumatische Querschnitt (sowohl hinsichtlich Art als auch Ausmaß der Myelonverletzung aus operativer, prognostischer, aber auch medikolegaler Indikation)

2. röntgenologischer bzw. CT-Verdacht auf instabile diskoligamentäre Verletzungen (mit oder ohne neurolog. Symptomatik), u. a. Abschätzung der HWS-Stabilität (Stifneck erforderlich?)

3. Patienten mit klinisch vermuteter diskoligamentärer Läsion, aber unauffälliger Radiografie (mit oder ohne neurolog. Symptomatik)

4. Nachweis bzw. Ausschluss einer Rückenmarksläsion bei vorhandener spinaler Neurologie, aber negativer Radiografie (z. B. SCIWORA bei Kindern)

5. Differenzierung traumatischer Myelonläsionen hinsichtlich intramedullärer Einblutungen (Letztere besitzen ein deutlich schlechteres finales Outcome)

6. Suche nach intraspinaler Raumforderung vor geschlossener Reposition bei zervikalen Facettengelenksluxationen (z. B. epidurale Hämatome, Bandscheibenextrusionen)

zunehmen, die eine effektivere Nutzung vorhandener Räumlichkeiten gestatten (z. B. Verbringung bodenständiger Versorgungseinheiten an und in die seitlichen Wände, Deckenlösungen für anästhesiologisches Equipment u. a.). Zudem würden es die Augsburger Verhältnisse erlauben, weitere und bereits vorhandene und nahegelegene Räumlichkeiten in die Polytraumaversorgung einzubeziehen und diese evtl. auch als Notfall-OP-Räume in der Schockraumumgebung aufzurüsten, wie es sie bislang in Augsburg in dieser Form nicht gibt (Trauma Operation Rooms, z. B. ausgestattet als Hybrid-OP, d. h. einer kombinierten CT-/Angiografie-/OP-Einheit [35]). 


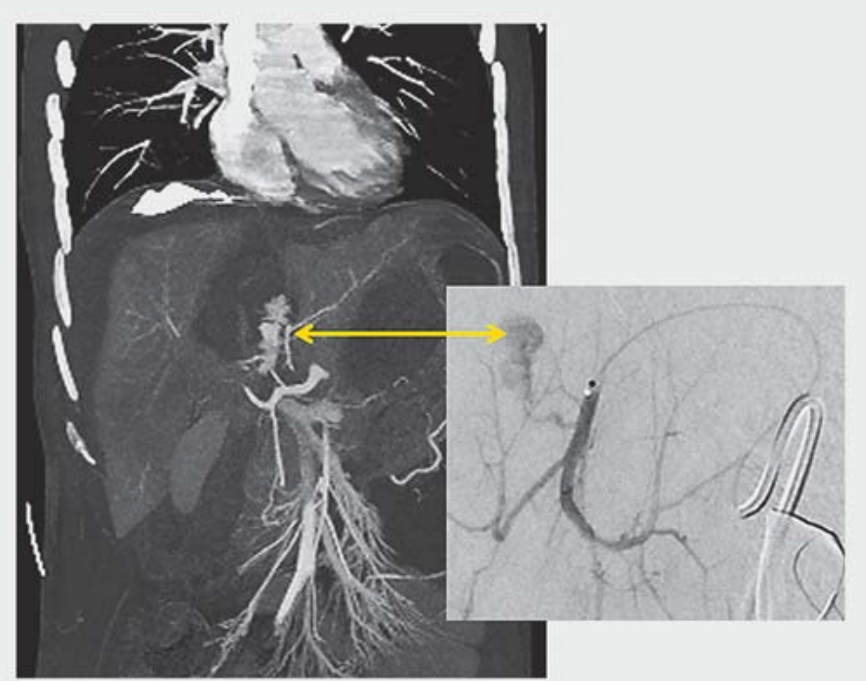

- Abb.11 Stumpfes Bauchtrauma mit aktiver Blutung aus einem Ast der A hepatica sinistra (CT-Angiografie; großes Bild): unmittelbar darauf erfolgte die transarterielle Embolisation (DAS; kleines Bild zeigt die präembolisatorische Situation).
Aus radiologischer Sicht wäre es - unter den gegebenen Umständen - vordringlich, zunächst einen zweiten modernen CT-Scanner innerhalb der Notfallradiologie zu installieren, der in erster Linie eine neuroradiologisch-diagnostische Abarbeitung u.a. der Schlaganfallpatienten verzögerungsfrei und vor allem unabhängig von der Polytraumaversorgung gewährleisten würde. Die Tatsache, dass - abgesehen von internistisch-chirurgischen Notfällen und Intensivpatienten des eigenen Hauses - sowohl die Polytraumaversorgung als auch die neurologische Schlaganfalldiagnostik im Wesentlichen auf ein und dasselbe CT zugreifen, stellt einen kritischen und potenziell auch gefährlichen diagnostischen Engpass dar. Zwar existieren klare Handlungsanweisungen und Absprachen unter den ärztlich Beteiligten, doch ist damit der diagnostische „Flaschenhals“ nur geregelt, aber nicht gelöst.

Unter dem Gesichtspunkt möglicher Massenanfälle Schwerverletzter stellt die logistisch umständliche Erreichbarkeit der weiteren CT-Räume ein Problem dar, was - wie schon diskutiert wurde - aufgrund baulicher Gegebenheiten nicht gelöst werden kann. Dies limitiert die optimale Auslastung vorhandener Geräteressourcen im MANV-Falle.

Die volldigitale Flachdetektorbildgebung in der konventionellen Radiografie stellt einen echten Fortschritt dar und hat damit einen zufriedenstellenden Stand erreicht, wohingegen das sonografische Equipment in der Notfallradiologie mit dem aktuellen Stand moderner Geräteausstattungen Schritt halten sollte, damit diagnose- und therapierelevante Entscheidungen rasch und vor allem mit hoher diagnostischer Sicherheit gefällt werden können. Letzteres gilt insbesondere für verletzte Kinder, denen man aus strahlenhygienischer Sicht eine Ganzkörper-CT - wann immer vertretbar - ersparen möchte (ALARAPrinzip).

Die MRT wird im Polytraumaszenario eine stets aufs Neue zu diskutierende diagnostische Modalität sein, da ihr Einsatz einen kreislaufstabilen Patienten im zeitunkritischen diagnostischen Fenster voraussetzt. Hierbei spielen also weniger die logistischen Gegebenheiten eine limitierende Rolle, sondern bilden vielmehr die Verletzungsschwere, die Art der Schädigung und der sich daraus ableitende diagnostische Zugewinn durch die MRT die entscheidenden Indikationen zur Durchführung einer MRT in der unmittelbaren Schockraumversorgung. Perspektivisch wird die MRT allerdings tatsächlich einen höheren Stellenwert bekommen als sie heute bereits besitzt.

Und nicht zuletzt wäre zum einen eine intelligente automatisierte Befundunterstützung mit voreingestellten Rekonstruktionsalgorithmen einschließlich sinnvoller 3-DDarstellungen, kurvilinearer Rippen- und Gefäßreformationen und anderer Nachbearbeitungs-Tools und zum anderen ein strukturiertes Befundungsschema unter Verwendung eindeutiger und gegenseitig anerkannter Begrifflichkeiten, Kategorisierungen und Klassifikationen begrüßenswert (Stichwort „künstliche Intelligenz“ [KI], artificial intelligence $[\mathrm{Al}]$ ), um einerseits der stetig steigenden „Bilderflut“ (bis zu 1500 Schnittbilder und mehr pro Schockraumfall) sinnvoll Herr zu werden und andererseits aber auch sprachlichen Missverständnissen, diagnostischen Fehlinterpretationen und zeitraubenden Nachfragen vorzubeugen bzw. diese zu reduzieren.

\section{Danksagung}

Der Autor dankt ausdrücklich Herrn Oberarzt Andreas Adam, Radiologe am Krankenhaus Friedberg-Aichach, für dessen kritische Manuskriptdurchsicht und wertvolle Ergänzungsvorschläge.

\section{Interessenkonflikt}

Der Autor gibt an, dass kein Interessenkonflikt besteht.

\section{Autorinnen/Autoren}

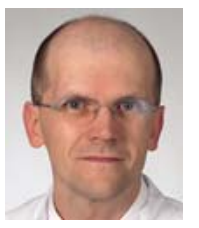

\section{Thomas Grieser}

Dr. med., Klinik für Diagnostische und Interventionelle Radiologie (Direktor: Prof. Dr. Th. Kroencke) Universitätsklinikum Augsburg 


\section{Korrespondenzadresse}

\section{Dr. med. Thomas Grieser}

Klinik für Diagnostische und Interventionelle Radiologie und Neuroradiologie

Universitätsklinikum Augsburg

Stenglinstraße 2

86156 Augsburg

Tel.: 0821/400-2441

Fax: 0821/4 00-3312

thomas.grieser@uk-augsburg.de

\section{Literatur}

[1] TraumaRegister DGU ${ }^{\circledR}$. Jahresbericht 2017. Im Internet: http://www.traumaregister-dgu.de/fileadmin/user_upload/ traumaregister-dgu.de/docs/Downloads/TR-DGUJahresbericht_2017.pdf; Stand: 31.07.2019

[2] Arbeitsgemeinschaft der Wissenschaftlichen Medizinischen Fachgesellschaften (AWMF). S3-Leitlinie Polytrauma/Schwerverletzten-Behandlung. AWMF Reg.-Nr. 012/019, Stand vom Juli 2016. Im Internet: https://www.awmf.org/uploads/ tx_szleitlinien/012-019I_S3_Polytrauma_SchwerverletztenBehandlung_2017-08.pdf; Stand: 31.07.2019

[3] Huber-Wagner S, Mand C, Ruchholtz S et al. Effect of the localisation of the $\mathrm{CT}$ scanner during trauma rescuscitation on survival - a retrospective, multicentre study. Injury 2014; 45 (Suppl. 3): S76-S82

[4] Fung Kon Jin PH, Goslings JC, Ponsen KJ et al. Assessment of a new trauma workflow concept implementing a sliding CT scanner in the trauma room: the effect on workup times. J Trauma 2008; 64: 1320-1326

[5] Scalea TM, Rodriguez A, Chiu WC et al. Focused Assessment with Sonography for Trauma (FAST): results from an international consensus conference. J Trauma 1999; 46: 466-472

[6] Ianniello S, Di Giacomo V, Sessa B et al. First-line sonographic diagnosis of pneumothorax in unstable major trauma: accuracy of e-FAST and comparison with multidetector computed tomography. Radiol Med 2014: 119: 674-680

[7] Braunschweig R, Reske S. Protokollempfehlungen der AG Bildgebende Verfahren des Bewegungsapparates (AG BVB) zur Ganzkörper-CT mehrfach verletzter bzw. polytraumatisierter Patienten. Fortschr Röntgenstr 2017; 189: 1002-1006

[8] Kulla M, Engelhardt M, Holsträter T et al. REBOA als additives Konzept zur Notfallthorakotomie beim Schwerstverletzten notwendig oder entbehrlich? Anästh Intensivmed 2018; 59: 562-573

[9] Reske SU, Braunschweig R, Reske AW et al. Polytrauma-Ganzkörper-CT: klinisch adaptierter Einsatz unterschiedlich gewichteter CT-Untersuchungsprotokolle. Fortschr Röntgenstr 2018; 190: 1141-1151

[10] Miele V, Addeo G, Cozzi D, Danti D, Bonasera L, Trinci M, Grassi R. Management of Polytrauma Patients. In: Miele V, Trinci M, eds. Diagnostic Imaging in Polytrauma Patients. Basel: Springer International Publishing; 2018: 1-28

[11] Briggs RH, Rowbotham AL, Johnstone AL et al. Provisional reporting of polytrauma CT by on-call radiology registrars. Is it safe? Clin Radiol 2010; 65: 616-622

[12] Quinn AC, Sinert R. What is the utility of the Focused Assessment with Sonography in Trauma (FAST) exam in penetrating torso trauma? Injury 2011; 42: 482-487

[13] Häuser H, Bohndorf K. Radiologisches Notfallmanagement polytraumatisierter Patienten. Radiologe 1998; 38: 637-644
[14] Sierink JC, Treskes K, Edwards M] et al. Immediate total-body CT scanning versus conventional imaging and selective CT scanning in patients with severe trauma (REACT-2): a randomized controlled trial. Lancet 2016; 388: 673-683

[15] Wurmb TE, Frühwald P, Hopfner W et al. Whole-body multislice computed tomography as the first line diagnostic tool in patients with multiple injuries: the focus on time. J Trauma 2009; 66: 658-665

[16] Topp T, Lefering R, Lopez CL et al. Radiologic diagnostic procedures in severely injured patients - is only whole-body multislice computed tomography the answer? Int J Emerg Med 2015; 8: 3. doi:10.1186/s12245-015-0053-8

[17] Mathews JD, Forsythe AV, Brady Z et al. Cancer risk in 680,000 people exposed to computed tomography scans in childhood or adolescence: data linkage study of 11 million Australians. BMJ 2013; 346: f2360

[18] Meltzer JA, Stone ME jr., Reddy SH et al. Association of wholebody computed tomography with mortality risk in children with blunt trauma. JAMA Pediatr 2018; 172: 542-549

[19] Linde F, Mani K, Juhlin C et al. Routine whole body CT of high energy trauma patients leads to excessive radiation exposure. Scandinavian J Trauma Resuscitation Emerg Med 2016; 24: 2. doi:10.1186/s13049-016-0199-2

[20] Munk RD, Strohm PC, Saueressig U et al. Effective dose estimation in whole-body multislice $\mathrm{CT}$ in paediatric trauma patients. Pediatr Radiol 2009; 39: 245-252

[21] Kellenberger C]. Bildgebung beim Kind - was ist speziell? Ther Umschau 2009; 66: 55-59

[22] Stengel D, Rademacher G, Ekkernkamp A et al. Emergency ultrasound-based algorithms for diagnosing blunt abdominal trauma. Cochrane Database Syst Rev 2015; (9): CD004446. doi:10.1002/14651858.CD004446.pub4

[23] Raptis CA, Mellnick VM, Raptis DA et al. Imaging of trauma in the pregnant patient. RadioGraphics 2014; 34: 748-763

[24] Mirza FG, Devine PC, Gaddipati S. Trauma in pregnancy: a systematic approach. Am J Perinatol 2010; 27: 579-586

[25] Jain V, Chari R, Maslovitz $S$ et al. Guidelines for the management of a pregnant trauma patient. J Obstet Gynaecol Can 2015; 37: 553-574

[26] Goldberg-Stein SA, Liu B, Hahn PF et al. Radiation dose management: part 2, estimating fetal radiation risk from $C T$ during pregnancy. AJR Am J Roentgenol 2012; 198: W352-W356

[27] Loose R, Wucherer M. Bildgebung in der Bildgebung. Radiopraxis 2017; 10: 131-138

[28] Parizel PM, van der Zijden T, Gaudino S et al. Trauma of the spine and spinal cord: imaging strategies. Eur Spine J 2010; 19 (Suppl. 1): S8-S17

[29] Kawakyu-O’Connor D, Bordia R, Nicola R. Magnetic resonance imaging of spinal emergencies. Magn Reson Imaging Clin N Am 2016; 24: 325-344

[30] Farrell CA, Hannon M, Lee LK. Pediatric spinal cord injury without radiographic abnormality in the era of advanced imaging. Curr Opin Pediatr 2017; 29: 286-290

[31] Huisman TA, Wagner MW, Bosemani T et al. Pediatric spinal trauma. J Neuroimaging 2015; 25: 337-353

[32] Frellesen C, Klein D, Tischendorf $P$ et al. Indication of whole body computed tomography in pediatric polytrauma patients - diagnostic potential of the Glasgow Coma Scale, the mechanism of injury and clinical examination. Eur J Radiol 2018; 105: $32-40$

[33] Pohlenz O, Bode PJ. The trauma emergency room: a concept for handling and imaging the polytrauma patient. Eur J Radiol 1996; 22: 2-6 
[34] Hiller JC, Tattersall D], Gleeson FV. Trainee reporting of computed tomography examinations: do they make mistakes and does it matter? Clin Radiol 2004; 59: 157-162

[35] Gemmette J], Chaudhary N, Pandey AS et al. Initial experience with a combined multidetector $\mathrm{CT}$ and biplane digital subtraction angiography suite with a single interactive table for the diagnosis and treatment of neurovascular disease. J Neurointerv Surg 2013; 5: 73-80

[36] Plumb AA, Grieve FM, Khan SH et al. Survey of hospital clinicians' preferences regarding the format of radiology reports. Clin Radiol 2009; 64: 386-396

[37] Thomson CB, Greaves I. Missed injury and the tertiary trauma survey. Injury 2008; 39: 107-114

[38] Clarke JR, Trooshin SZ, Doshi PJ et al. Time of laparotomy for intra-abdominal bleeding from trauma does effect survival for delays up to 90 minutes. J Trauma 2002; 52: 420-425

[39] Kumar Y, Hayashi D. Role of magnetic resonance imaging in acute spinal trauma: a pictorial review. BMC Musculoskeletal Disord 2016; 17: 310

[40] Bozzo A, Marcoux J, Radhakrishna M et al. The role of magnetic resonance imaging in the management of acute spinal cord injury. J Neurotrauma 2011; 28: 1401-1411

[41] Avery LE, Stahlfeld KR, Corcos AC et al. Evolving role of endovascular techniques for trauma vascular injury: a changing landscape? J Trauma Acute Care Surg 2012; 72: 41-46

[42] Velmahos GC, Toutouzas KG, Vassiliu P et al. A prospective study on the safety and efficacy of angiographic embolization for pelvic and visceral injuries. J Trauma 2002; 53: 303-308

[43] Royal College of Radiologists. Standards of practice and guidance for trauma radiology in severely injured patients. London: Royal College of Radiologists; 2001

[44] Cowley R, Hudson F, Scanlan E et al. An economical and proved helicopter program for transporting the emergency critically ill and injured patient in Maryland. J Trauma 1973; 13: 10291038

[45] Berger E. Nothing gold can stay? EMS crashes, lack of evidence bringing the golden hour concept under new scrutiny. Ann Emerg Med 2010; 56: A17-A19
[46] Kleber C, Lefering R, Kleber A] et al. Rettungszeit und Überleben von Schwerverletzten in Deutschland. Unfallchirurg 2013; 116: $345-350$

[47] Deutsche Gesellschaft für Medizinische Physik e.V. DGMPBericht Nr.7, 2002: Pränatale Strahlenexposition aus medizinischer Indikation. Dosisermittlung, Folgerungen für den Arzt und Schwangere. Im Internet: https://www.dgmp.de/ media/document/205/bericht7-neuauflage2002.pdf; Stand: 31.07.2019

[48] Frellesen C, Boettcher M, Wichmann JL et al. Evaluation of a dual-room sliding gantry CT concept for workflow optimization in polytrauma and regular in- and outpatient management. Eur J Radiol 2015; 84: 117-122

[49] Kahn J, Grupp U, Kaul D et al. Computed tomography in trauma patients using iterative reconstruction: reducing radiation exposure without loss of image quality. Acta Radiol 2016; 57: 362-369

[50] Soto JA, Lucey BC, Stuhlfaut JW et al. Use of 3D imaging in CT of the acute trauma patient: impact of a PACS-based software package. Emerg Radiol 2005; 11: 173-176

[51] Saigal K, Winokur RS, Finden S et al. Use of three-dimensional computerized tomography reconstruction in complex facial trauma. Facial Plast Surg 2005; 21: 214-220

[52] Khung S, Masset P, Duhamel A et al. Automated 3D rendering of ribs in 110 polytrauma patients: strengths and limitations. Acad Radiol 2017; 24: 146-152

[53] Wolfschmidt F, Dierks A, Wumb T et al. Innerklinisches Traumamanagement - Diagnostik im Schockraum. Anästhesiol Intensivmed Notfallmed Schmerzther 2014; 49: 536-543

[54] Harvey J], West ATH. The right scan, for the right patient, at the right time: The reorganization of major trauma service provision in England and its implication for radiologists. Clin Radiol 2013; 68: 871-886

\section{Bibliografie}

DOI https://doi.org/10.1055/a-0986-3303

Online-publiziert 08.01.2020 | OP-JOURNAL 2020; 36: 60-74 (c) Georg Thieme Verlag KG Stuttgart · New York ISSN 0178-1715 